\title{
Thyrocalcitonin assay: comparison of two immuno- radiometric KITS, IRMA hCT cisbio international vs IRMA hCT beckman-coulter
}

\begin{abstract}
Problematic: CT is a useful serum marker for the diagnosis and follow-up of CMT. However the values of $\mathrm{CT}$ are different, resulting in difficulties in interpreting the results. In this study, we compared two radioimmunoassay kits for CT assay.

Patients and methods: CT was measured in 66 patients using two radioimmunoassay kits: IRMA hCT Cisbio International which is the gold standard and IRMA hCT BeckmanCoulter. A comparison of the average values, a correlation test, a Bland-Altman diagram, a Passing Bablok linearity test and ROC curve were performed.

Results: There is a good correlation between the 2 kits, the problem of dispersion in the high CT values observed with the BECKMAN-COULTER kit is not a major problem since the diagnosis of the pathology of $\mathrm{C}$ cells is already posed, and in definitive BECKMANCoulter kit can represent an alternative to CISBIO International IRMA kit.
\end{abstract}

Volume 6 Issue I - 2018

\author{
Sidi Mohammed Meghelli, Nour El Houda \\ Khelil, Necib Berber \\ Department of Nuclear Medicine, University Hospital of \\ Tlemcen, Algeria
}

Correspondence: Nour El houda Khelil, Department of Nuclear Medicine, In-vitro Exploration Unit, University Hospital of Tlemcen, Faculty of Medicine Dr. Benzerdjeb Benaouda Tlemcen, 12, Rue HAMRI Ahmed, B.P 123, Tlemcen, Algeria, Tel 002I355649906I,Email khelilhouda@yahoo.fr

Received: November 10, 2017 | Published: January 02, 2018

Keywords: calcitonin, immunoradiometric assays, medullary thyroid carcinomas

\section{Introduction}

Thyroid cancers represent $3-4 \%$ of all human tumors. ${ }^{1}$ Nevertheless, it is the most common endocrine neoplasia. Its incidence has increased over the past two decades. ${ }^{2}$ It can be categorized into different histotypes: papillary carcinoma of the thyroid (CPT), follicular thyroid carcinoma (CFT) and anaplastic thyroid carcinoma (ATC), all derived from follicular cells and medullary carcinoma of the thyroid (CMT), which comes from parafollicular C cells. ${ }^{1}$

CMT represents $5-10 \%$ of thyroid cancers, ${ }^{3}$ it retains the ability to secrete CT, who is a marker for diagnosis and follow-up. CT is a 32 amino acids protein. ${ }^{4}$ It is the leader of a large peptide family: CT gene-related peptides I and II (CGRP-I and II), adrenomedullin, amylin. Its secretion is regulated by the serum calcium levels; it has a hypocalcemic and hypophosphatemic action. ${ }^{4}$

To ensure optimal management of CMT, biological explorations must be precise, combined with rigorous interpretation. The CT assay is nowadays more and more often performed for the detection of CMT in the context of thyroid nodular pathology. ${ }^{5}$ It is a very sensitive but not very specific tumor marker. In fact, hypercalcitoninemia can also be related to other pathologies, such as Hashimoto's thyroiditis, renal failure, endocrine tumors other than CMT, smoking and taking proton pump inhibitors. ${ }^{6}$ Important progress has been made in the field of serum CT immunoradiometric assays, all based on antigen-antibody reactions with specificities and standards specific to each kit. ${ }^{7}$

As part of the follow-up of patients with CMT, the reference kit of the CT assay is IRMA hCT Cisbio international. ${ }^{3}$ It has been used in the nuclear medicine department of Tlemcen hospital for several years. Recently, another kit using the same principle of CT immunoassay and less expensive than that of Cisbio appeared on the market, that of the supplier Beckman-Coulter. In order to reduce the cost of treating our patients with CMT and to test Beckman-Coulter's new radioimmunometric kit, we compared the $\mathrm{CT}$ values obtained with the latter to the IRMA hCT Cisbio International kit takes in this work as Gold Standard.

\section{Patients and methods}

The study is conducted in the nuclear medicine department of Tlemcen University Hospital over a period of 08 months between September 2016 and May 2017. Were included, patients with suspicion of CMT, patients followed for CMT already operated and subjects who are presumed healthy after preliminary interrogation to rule out any cause of functional hypercalcitoninemia. Patients who were not fasting during the sampling, those with tobacco and or alcohol consumption, pregnant women and patient's dialysis were excluded from the study.

All subjects were informed of the study protocol and oral, free and informed consent was obtained. The samples of eligibly patients were taken in dry tubes; the sera were aliquoted and immediately frozen at $-20^{\circ} \mathrm{C}$. The sera underwent a single freeze/thaw cycle the same day of the assay, at ambient temperature. On the same serum divided into several aliquots, were dosed the CT simultaneously and by the same manipulator with the IRMA hCT Cisbio International kit and IRMA hCT Beckman-Coulter kit. An imprecision test was evaluated for all the samples on the 02 kits by making 03 readings/day for 03 days for each of the kits (Table 1).

The CT assay is manual, radioimmunometric or sandwich type, using antibodies directed against two different epitopes of the CT molecule, and reacting without competition. The sample is first brought into contact with the first antibody. After incubation and aspiration, the second monoclonal antibody is added, labeled with 125I. After incubation and aspiration, bound radioactivity is then measured. The amount of radioactivity fixed is directly proportional to the CT concentration of the sample measured by the gamma counter which is a tool for measuring the intensity of gamma radiation essentially used in the field of bioanalysis.

The readings were performed by gamma counter with 05 detectors of Perkin-Elmer brand. To avoid any risk of overestimation of the concentration of $\mathrm{CT}$ due to radioactive contamination, the readings were made on the same tube holders for the 02 kits. All sera, standards and controls were assayed in duplicate. The control sera provided 
by the 02 kits, were used in each assay series with respectively 01 only control level for Beckman Coulter kit and 02 levels for CisbioInternational kit, they were positioned randomly within the assay series.

The statistical analysis was done by SPSS software version 21 and XLSTAT software. Pearson's bivariate parametric test was applied to assess the degree of correlation (r) of the quantitative variables. The Altman Bland test was used to evaluate the linearity of the values obtained on the 02 kits as well as the Passing-Bablok regression line. The sensitivity (Se) and specificity ( $\mathrm{Sp}$ ) of the IRMA hCT BeckmanCoulter kit at $10 \mathrm{pg} / \mathrm{ml}$ (reference threshold of the IRMA hCT Cisbio International kit taken as gold standard) was evaluated using the ROC curve.

Table I Technical characteristics of the 02 kits (IRMA hCT Cisbio International and IRMA hCT Beckman-Coulter)

\begin{tabular}{lll}
\hline Characteristics & IRMA hCT cisbio international & IRMA hCT beckman-coulter \\
\hline Principle & Sandwich & Sandwich \\
& Coated tubes Anti-hCT monoclonal Antibody & Coated tubes Anti-hCT monoclonal Antibody \\
Monoclonal Antibody & $125 /$ anti-hCT monoclonal antibody & $125 /$ anti-hCT monoclonal antibody \\
& $11,6+/-1.74 \mathrm{pg} / \mathrm{mL}$ & $41.8+/-10.5 \mathrm{pg} / \mathrm{mL}$ \\
Control & 05 calibrators & $95.0+/-14.4 \mathrm{pg} / \mathrm{mL}$ \\
& 7.9 & Calibrator $0+05$ calibrators \\
& 31 & 7.6 \\
Calibrators & 110 & 29 \\
& 710 & 50 \\
Working range & 1400 & 159 \\
Incubation time & {$[0-1530] \mathrm{pg} / \mathrm{mL}$} & 740 \\
Analytical sensitivity & $20-24 \mathrm{~h}$ to room temperature $\left(18-25^{\circ} \mathrm{C}\right)$. & {$[0-674] \mathrm{pg} / \mathrm{mL}$} \\
Functional sensitivity & $1,5 \mathrm{pg} / \mathrm{mL}$. & $18 \mathrm{~h}$ to room temperature $\left(18-25^{\circ} \mathrm{C}\right)$ \\
Normal values & $4 \mathrm{pg} / \mathrm{mL}$ & $0.9 \mathrm{pg} / \mathrm{mL}$ \\
Local cost & $<10 \mathrm{pg} / \mathrm{ml}$ & No defined \\
\hline
\end{tabular}

\section{Results}

Sixty-six samples belonging to 53 women and 13 men were analyzed. The average age of participants was 51.23years for women (20-79years) and 49.61years for men (25-75years). The average of CT was higher in men with $2 \mathrm{kits}$, it is $40.53 \mathrm{pg} / \mathrm{ml}$ in women and $102.39 \mathrm{pg} / \mathrm{ml}$ in men with the IRMA hCT Cisbio International kit and $108.41 \mathrm{pg} / \mathrm{ml}$ in women and $189.62 \mathrm{pg} / \mathrm{ml}$ in men with the IRMA hCT Beckman-Coulter kit. The box plot (Figure1) illustrates the distribution of CT concentrations obtained with the 02 kits.

Taking into account the threshold of positivity of the reference kit $(10 \mathrm{pg} / \mathrm{ml})$, it appears that $21 \%(14 / 66)$ of the subjects have a positive CT rate for the Beckman kit compared to only $9 \%(6 / 66)$ for the Cisbio kit. Moreover, there is a positive and significant correlation between the CT values obtained on the IRMA hCT Cisbio International kit and that of IRMA hCT Beckman-Coulter $(\mathrm{r}=0.84, \mathrm{p}<0.05)$. The repeatability test revealed a $\mathrm{CV}<10 \%$ for the 02 kits. The Altman Bland diagram revealed no difference in means between the CT values obtained with the 02 kits $(\mathrm{P}$-value $=0.23>0.05)$, (Figure 2$)$. The passing-Bablok regression line showed a good linearity of most of the CT values between the 02 kits with a p-value at $0.44>0.05$ (Figure 3 ). The ROC curve returned $83 \%$ sensitivity and $98 \%$ specificity for the IRMA hCT Beckman-Coulter kit. The CT threshold corresponding to these diagnostic performances is $13.69 \mu \mathrm{g} / \mathrm{ml}$ (area under the curve: 0.872 ).

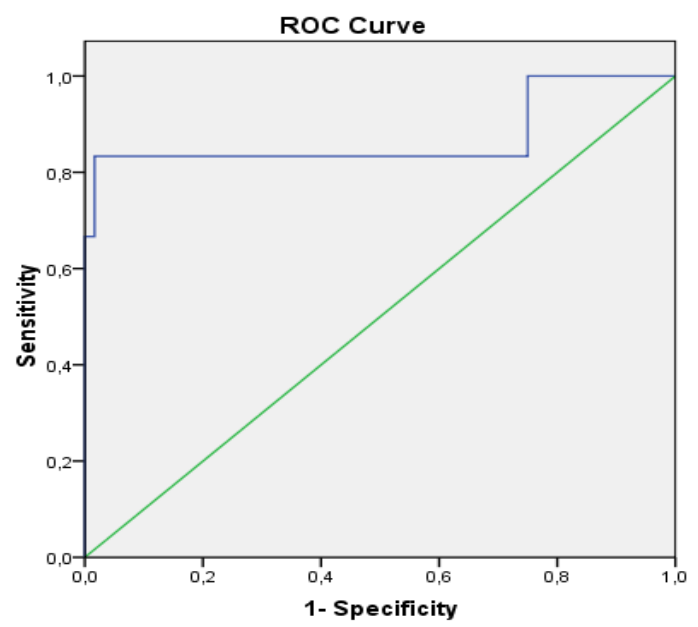




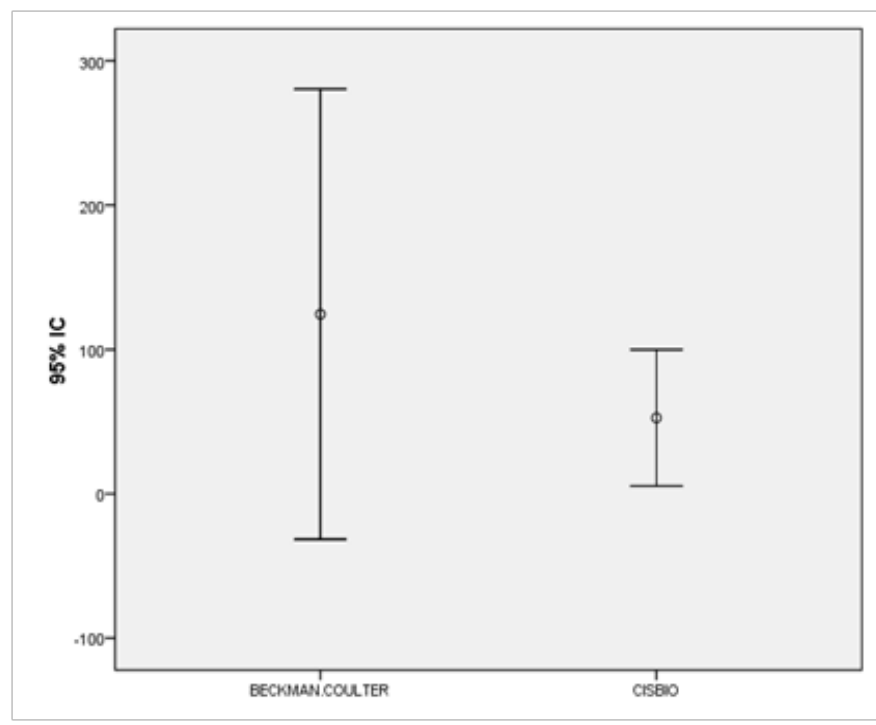

Figure I Distribution of CT values with the 02 kits.

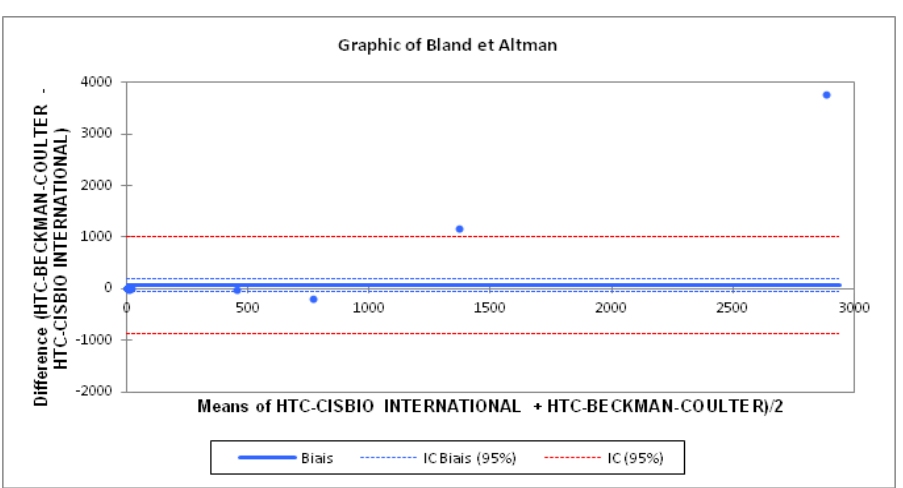

Figure 2 Comparison of mean differences by the Bland and Altman test.

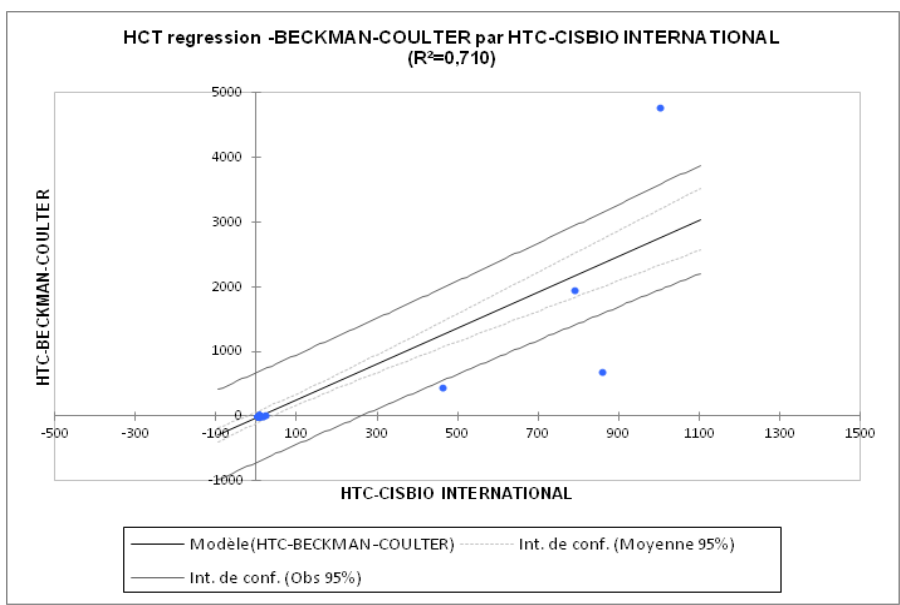

Figure 3 Passin-Bablok regression line.

\section{Discussion}

Medullary thyroid cancer is a rare but potentially serious pathology. Serum CT is the key to diagnosis and monitoring. It is a sensitive marker but not very specific especially for the moderate values (30 to $50 \mathrm{pg} / \mathrm{ml}),{ }^{8}$ in these situations the pentagastrine stimulation test found all its interest. The absence of pentagastrin should lead us to rethink diagnostic and surveillance procedures. ${ }^{8}$ As a result, CT dosing kits need to be more and more efficient for optimal patient management.

The immunoradiometric reference assay currently used in our service (IRMA hCT Cisbio-International) is based on a sandwich method using two monoclonal antibodies directed against mature monomeric $\mathrm{CT}^{4}{ }^{4}$ In order to introduce an alternative to the latter, and evaluating the cost-effectiveness ratio, we compared the results obtained with the reference kit to those obtained with the Beckman Coulter kit. In this work, a sample of 66 sera was simultaneously assayed by the IRMA hCT Cisbio International Kit and IRMA hCT Beckman-Coulter Kit.

It seems appropriate to start our discussion by explaining the limits of our study that are: the small size of our sample and a confirmed case of CMT because the rarity of the pathology, the duration recruitment and the monocentric nature of the study. The descriptive analysis of the sample shows a clear female predominance $(80 \%)$ with a sex ratio of 0.24: 1 man for every 4women, and the average age of men/women is 49 and 51 respectively.

The average CT obtained in men with the Cisbio kit and the Beckman Coulter kit is significantly higher than that of women, this could be explained by the fact that the $\mathrm{C}$ cell contingent is significantly higher in men. ${ }^{9}$ A study done on an autopsy series concludes that the number of $\mathrm{C}$ cells is multiplied by 2 in men. ${ }^{10}$

The notion of tobacco and alcohol intake cannot explain this difference in $\mathrm{CT}$ means between men/women; it was eliminated by the questioning before inclusion. BMI was not calculated for our patients. In the D'herbomez study of 287 subjects, the correlation between CT, age and BMI was well established, particularly in men. ${ }^{9}$

Regarding the $\mathrm{CT}$ values and taking into consideration the threshold value of $10 \mathrm{pg} / \mathrm{mL}$ (that of the reference kit), the number of patients positive with IRMA hCT Beckman-Coulter (14/66) was twice that of from IRMA hCT Cisbio international (6/66), this could be due to a random error in sample taking during the assay, or also because the functional sensitivity is quite low for the reference kit $(4 \mathrm{pg} / \mathrm{ml})$ while undefined for the Beckman Coulter Kit.

In order to evaluate the intra-serie precision (repeatability), the serum samples were read consecutively within the same series for 3 days for each of the kits. With a total of 9 readings, the coefficients of variation gave values of $<10 \%$ for IRMA hCT Cisbio international and IRMA hCT Beckman-Coulter, thus testifying to the non-drifting of values over all readings.

The bivariate Pearson correlation test revealed a strong and significantly correlation between the CT values obtained on the IRMA hCT Cisbio international kit and that of hTC-Beckman ( $\mathrm{r}=0.84$, $\mathrm{p}<0,05$ ). This correlation is not surprising, given that these two kits use the same principle of sandwich-type immunoassay and the same type of revelation antibody.

The distribution of the CT values obtained on the 02 kits, showed a wider dispersion of values for IRMA hCT Beckman-Coulter than for IRMA hCT Cisbio international, this could be due to the absence of parity between the standards of the two kits. Indeed, the standards provided with the IRMA hCT Beckman-Coulter kit to establish the calibration range does not properly cover the high concentration zone, with notably a large difference from the 3 rd (theoretical concentration $50 \mathrm{pg} / \mathrm{mL}) 4^{\text {th }}$ (concentration theoretical $159 \mu \mathrm{g} / \mathrm{ml}$ ) and $5^{\text {th }}$ standard (theoretical concentration $740 \mu \mathrm{g} / \mathrm{ml}$ ). 
In order to verify the linearity of the values obtained in each of the kits, the Passing and Bablok rule (bilateral test) was used, giving a bilateral p-value of $0.44(>0.05)$. This concluded a good linearity of the values except for 04 samples with high concentrations.

The comparison of the mean differences of pair-matched CTs on the 02 kits was performed by the Bland-altman test, which found a significance factor $\mathrm{p}=0.23>0.05$, indicating the absence of a significant difference between the two means, with the exception of samples with high concentrations corresponding to CMT cases. This is consistent with an Italian study of 35 confirmed CMT cases comparing 04 assay kits all having the same immunometric assay principle (IRMA hCT Cisbio International , US CT (Bio source), Ultra Sensitive Calcitonin (DSL) and Calcitonin Assay (Sc antibody) having found a low correlation between the values obtained on the 04 kits. ${ }^{11}$

A ROC curve has been established to judge the performance of the IRMA hCT Beckman-Coulter kit against the reference level of 10pg $/ \mathrm{ml}$. the area under the curve is 0.87 , the test is then significant in the majority of cases.

The diagnostic value of a test is all the more reliable as it is both sensitive and specific. As a result, we have estimated from the coordinates of the ROC curve that for IRMA hCT Beckman-Coulter the threshold that gives the best compromise between the sensitivity $(83 \%)$ and the specificity $(98 \%)$ is: $13.69 \mathrm{pg} / \mathrm{ml}$ (higher than IRMA hCT Cisbio International).

The analysis of the performances mentioned by the suppliers highlights a greater analytical sensitivity for the IRMA hCT Beckman-Coulter kit $(0.9 \mathrm{pg} / \mathrm{mL}$ VS $1.5 \mathrm{pg} / \mathrm{mL}$ for IRMA hCT Cisbio International), it is in theory, more efficient in the low values of CT. This difference in sensitivity between the two kits in the very low values does not really have any significant consequence in the clinical level, since the results are interpreted in relation to a decision threshold equal to $10 \mu \mathrm{g} / \mathrm{mL}$ for IRMA hCT Cisbio International (reference kit).

With regard to these results, the IRMA hCT Beckman-Coulter kit would be a good alternative to the IRMA hCT Cisbio International kit especially since it is at our level 2.5 times less expensive, however it is recommended, for the follow-up of the patients to always determine the $\mathrm{CT}$ rates within the same lab with the same assay kit, and to inform the clinician of any changes.

However, it seems interesting to work in the future, on a larger sample comprising only confirmed CMT cases to truly evaluate the effectiveness of the IRMA hCT Beckman-Coulter kit, while involving other automated dosing kits which would eliminate the risk of human error.

\section{Acknowledgment}

None.

\section{Conflicts of interest}

The authors declare that there is no conflict of interest.

\section{References}

1. Pellegriti G, Frasca F, Regalbuto C, et al. Worldwide increasing incidence of thyroid cancer:update on epidemiology and risk factors. $J$ Cancer Epidemiol. 2013;2013:965212.

2. Romei C, Ciampi R, Elisei R. A comprehensive overview of the role of the RET proto-oncogene in thyroid carcinoma. Nat Rev Endocrinol. 2016;12(4):192-202.

3. Nicolli-Sire P, Conte-Devolx B. Cancer medullaire of thyroid. Enccyclopedie Orphanet. 2007.

4. Modigliani E. Le cancer médullaire de la thyroïde. Rev Med Interne. 1999;20(6):490-503.

5. Wémeau JL, Sadoul JL, d'Herbomez M, et al. Recommandations de la Société française d'endocrinologie pour la prise en charge des nodules thyroïdiens. 2011

6. Niccoli P, Wion-Barbot N, Caron P, et al. Interest of routine measurement of serum calcitonin (CT): study in a large series of thyroidectomized patients. J Clin Endocrinol Metab. 1997;82(2):338-341.

7. Censi S, Cavedon E, Fernando SW, et al. Calcitonin measurement and immunoassay interference:a case report and literature review. Clin Chem Lab Med. 2016;54(12):1861-1870.

8. Laboureau SS, Rohmer V. Le carcinome médullaire de la thyroid en 2012:What is the base calcitonin in the absence of a stimulation tes for diagnosis, prognostic and le traitement? Mise au point cliniques d'Endocrinologie. 2012.

9. D’Herbomez M, Caron P, Bauters C, et al. Reference range of serum calcitonin levels in humans:influence of calcitonin assays, sex, age and cigarette smoking. Eur J Endocrinol. 2007;157(6):749-755.

10. Engelbach M, Gorges R, Forst T, et al. Improved diagnostic methods in the follow-up of medullary thyroid carcinoma by highly specific calcitonin measurements. J Clin Endocrinol Metab. 2000;85(5):1890-1894.

11. Martinetti A, Seregni E, Ferrari L, et al. Evaluation of circulating calcitonin:analytical aspects. Tumori. 2003;89(5):566-568 